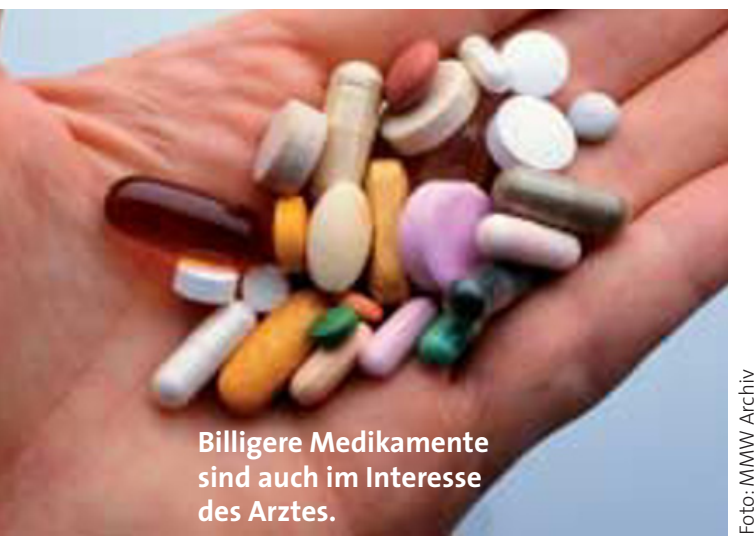

Die AOK hat mit elf weltweit operierenden Generikafirmen Rabattverträge für 2007 abgeschlossen. Davon profitieren Patienten, Apotheker und Ärzte. Die Liste der rabattierten Arzneimittel ist jetzt auch im Internet abrufbar.

- Ärzte, die preisreduzierte Arzneimittel verordnen, profitieren erstens davon, indem sie ihr Arzneimittelbudget nicht so schnell ausschöpfen, und zweitens dadurch, dass alle rabattierten Arz-

\title{
Kostensenkung
}

\section{Jetzt online: Liste reduzierter AOK-Arzneimittel}

neimittel nicht unter die Malus-Regelung fallen. Darüber hinaus haben einige AOKs wie etwa die AOK Baden-Württemberg Verträge mit bestimmten Kassenärztlichen Vereinigungen getroffen und sich verpflichtet, den Ärzten den Beratungsaufwand, den die Medikamentenumstellung mit sich bringt, zu vergüten (siehe auch Bericht in der MMW Nr. 11/2007, S. 54 und S. 55).

Die Preisnachlässe gibt es bis dato für 43 Wirkstoffe und Wirkstoffgruppen. Ab sofort finden AOK-Vertragspartner diese Arzneimittel im Internet unter www. aok-gesundheitspartner.de/bundesverband/arztundpraxis/arzneimittel/rabatt/ index.html. Die Suche ist komfortabel und kann über den Präparate-, Wirkstoff- und Firmennamen erfolgen. Die AOK aktualisiert die Daten 14-täglich.
Bundesweit rechnen die 16 AOKs durch die Rabattverträge mit Einsparungen im zweistelligen Millionenbereich. „Das ist angesichts der für 2007 zu erwartenden Mehrausgaben für Medikamente eine dringend notwendige Entlastung“, konstatiert der stellvertretende Vorsitzende des AOK-Bundesverbandes Johann Magnus von Stackelberg. Durch die Anhebung der Mehrwertsteuer auch für Arzneimittel und weitere Effekte sei im laufenden Jahr mit um sieben Prozent höheren Ausgaben für Medikamente zu rechnen. Bereits im Januar 2007 hat die gesetzliche Krankenversicherung 2,032 Milliarden Euro für Arzneimittel ausgegeben. Das waren rund 89 Millionen Euro oder 4,6\% mehr als im Januar 2006.

$B R$.

\section{Aktueller Abrechnungstipp}

\section{Wundversorgung bei Berufsunfällen}

Die ärztliche Versorgung bei Berufsunfällen hat ihre Besonderheiten - auch die Abrechnung. Dr. Roman Machens sagt, worauf es ankommt.

— Um es uns Ärzten nicht zu leicht zu machen, gibt es eine eigene UV-GOÄ 2001 nur für die Berufsgenossenschaften. Von den Honoraren abgesehen entspricht diese noch der "alten" GOÄ. Besorgen Sie sich ein entsprechendes Büchlein oder laden Sie die Dokumente kostenlos herunter unter www.KBV.de/Rechtsquellen/2350.html.

Normale kleine Verletzungen rechnen Sie z.B. ab mit

- Beratung $1=6,21$ Euro,

- Verband 200 = 3,24 Euro plus immer gleiche Kosten 1,30 Euro,

- Wundversorgung 2000 (auch mit Steristrips) $=4,83$ Euro.

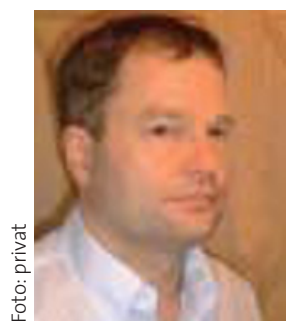

Dr. med. Roman Machens setzt sich intensiv mit Abrechnungsfragen auseinander.

Erfreulicherweise gibt es hier keine Budgetierung - Sie können also die indizierte Anzahl von Terminen veranlassen. Sie dürfen zugleich Kassenleistungen wegen anderer Diagnosen erbringen. Es liegen juristisch klar getrennte Behandlungsverhältnisse vor. Sie verbessern aber Ihre Wirtschaftlichkeit, wenn Sie z.B. Termine zur Wundkontrolle und Termine für Gesundheitsuntersuchungen zusammenlegen. Sie müssen lediglich für sich klar trennen, wann Sie in welchem Bereich tätig sind - BG oder EBM. Dem Patienten ist es fast gleichgültig, wie Sie abrechnen, solange es ihn nichts kostet.

Besondere Therapieverfahren wie Vitamininjektionen, Farblicht oder Laser zur Verbesserung der Wundheilung akzeptieren die Berufsgenossenschaften typischerweise nicht, aber andererseits sprechen sie bisher auch keine Regresse aus, wenn Sie rezeptfreie Medikamente verordnen. Nutzen Sie also alle solchen Gelegenheiten, und verordnen Sie bei entsprechender Indikation auf BG-Rezept

- Salben,

- wundheilungsfördernde Medikamente,

- Homöopathika.

DR. ROMAN MACHENS 